Check for updates

Cite this: RSC Adv., 2018, 8, 25342

\title{
Bi-functional heterogeneous catalysts for carbon dioxide conversion: enhanced performances at low temperature $\uparrow$
}

\author{
Adrien Comès, ${ }^{a}$ Xavier Collard, ${ }^{a}$ Luca Fusaro, ${ }^{a}$ Luciano Atzori, ${ }^{b}$ M. Giorgia Cutrufello ${ }^{b}$ \\ and Carmela Aprile (D) *a
}

Novel heterogeneous bi-functional catalysts bearing tin or zinc inserted as single sites within the silica architecture acting as acid centres and decorated with imidazolium moieties as the nucleophile source were successfully synthesized. The materials were extensively characterized via various techniques including $\mathrm{N}_{2}$ physisorption, solid state nuclear magnetic resonance, $\mathrm{X}$-ray photoelectron spectroscopy, transmission electron microscopy and adsorption microcalorimetry. The solids were tested as catalysts for the conversion of carbon dioxide, selecting the synthesis of styrene carbonate as the target reaction. Both materials exhibited improved performances compared to the analogous solids functionalized with the sole imidazolium salt as well as to other materials reported in the literature. The Sn-based catalyst displayed excellent conversion also in the presence of various epoxides. In all experiments the bifunctional solid allowed reducing the reaction temperature below $150{ }^{\circ} \mathrm{C}$. In the presence of glycidol the temperature was decreased down to $30^{\circ} \mathrm{C}$. The short synthesis protocol of the heterogeneous catalysts, together with the $100 \%$ atom economy of the target reaction and the low reaction temperature, make the entire process highly sustainable. Moreover, the Sn-based catalyst was stable under the selected reaction conditions and reusable for multiple catalytic cycles.

Received 6th May 2018

Accepted 6th July 2018

DOI: $10.1039 / c 8 r a 03878 c$

rsc.li/rsc-advances can find application as aprotic polar solvents, electrolytes for batteries and raw-materials for the preparation of polycarbonates. $^{3-7}$ Amongst the catalysts able to efficiently convert $\mathrm{CO}_{2}$ and epoxides into the corresponding cyclic carbonates, ammonium, phosphonium and imidazolium salts deserve to be mentioned. $^{8}$ To achieve an easy recovery from the reaction mixture and reuse of the catalyst for multiple cycles, most of these salts were also heterogenized ${ }^{9}$ on different supports. Silica-based porous materials, ${ }^{10,11}$ polymers, ${ }^{12,13}$ single as well as multi-walled carbon nanotubes, ${ }^{14-16}$ and more recently carbon nanohorns ${ }^{17}$ among others were already selected for this purpose. The mechanism of the reaction is well known and was already reported in various publications. ${ }^{6}$ The reaction is initiated by the attack of a nucleophile (usually represented by a halide) to the $\mathrm{C} 1$ or the $\mathrm{C} 2$ carbon, depending on the substituents, of the epoxide via a $\mathrm{S}_{\mathrm{N}} 2$ mechanism. The ring opening is followed by $\mathrm{CO}_{2}$ insertion with generation of a cyclic carbonate. However, when a catalyst such as ammonium or imidazolium salts is used in the absence of a co-catalyst, high temperatures (usually higher than $125^{\circ} \mathrm{C}$ ) are needed..$^{18}$

The high energy input required for the transformation of carbon dioxide can be further reduced employing a species (herein called co-catalyst) able to activate the three-membered ring via coordination of the oxygen with a metal centre acting as Lewis acid or through formation of hydrogen bond. ${ }^{1,18}$ The first strategy is largely exploited and widely described in the 
literature. A series of organometallic complexes was already reported by different authors. Various salen-, phthalocyanine-, porphyrins-based complexes bearing $\mathrm{Al}, \mathrm{Co}, \mathrm{Cr}$ or $\mathrm{Zn}$ as metal centres were successfully used for this purpose. ${ }^{\mathbf{1}}$ Tangestaninejad and co-workers also proposed a Sn-based porphyrin complex which displayed improved performances due to an increased coordination strength. ${ }^{19}$ The use of molecules displaying two or three metal centres was also suggested. Kleij and co-workers designed a tri-nuclear Zn-based complex exhibiting bi-functional properties. ${ }^{20}$ The use of various metal-organic frameworks (MOF) in combination with ammonium or imidazolium salts was also reported. ${ }^{21-23}$ The majority of the previously reported examples refer to homogeneous catalysts or systems in which one of the two components (catalyst or cocatalyst) was heterogenized. In the broad list of contributions addressing the synthesis of cyclic carbonates from $\mathrm{CO}_{2}$, only a minor number of publications describe the use of a heterogeneous bi-functional catalyst in which both catalyst and Lewis acid co-catalyst are heterogenized on one support and even fewer illustrate the use of inorganic metal centres acting as Lewis acid sites.

Herein, the design of two novel heterogeneous bi-functional catalysts presenting both a metal centre ( $\mathrm{Zn}$ or $\mathrm{Sn}$ ) able to bring acid properties and the nucleophile required to promote the opening of the epoxide was successfully achieved. The synthesis procedure involved the initial preparation of a porous support bearing a metal element inserted as single sites within the silica architecture followed by the grafting of an imidazolium salt. The solids were extensively characterized via various techniques including $\mathrm{N}_{2}$ physisorption, X-ray diffraction, ${ }^{13} \mathrm{C}$ and ${ }^{29} \mathrm{Si}$ solid state nuclear magnetic resonance, transmission electron microscopy, combustion chemical analysis and inductively coupled plasma optical emission spectroscopy. The insertion of $\mathrm{Sn}$ and $\mathrm{Zn}$ as single sites within the silica structure was proved via solid state ${ }^{119} \mathrm{Sn}$ nuclear magnetic resonance and X-ray photoelectron spectroscopy respectively, while the acid properties of the solids were addressed via adsorption microcalorimetry.

The materials were tested under solvent-free conditions in a batch reactor selecting the chemical fixation of $\mathrm{CO}_{2}$ into styrene oxide as target reaction. Both $\mathrm{Sn}$ - and $\mathrm{Zn}$-based solids displayed improved performances compared to the analogous silica catalyst functionalized with the sole imidazolium salt as well as to other solids reported in the literature. The versatility of the Sn-based catalyst was also tested with a series of epoxides. In all experiments, the bi-functional solid allowed reducing the reaction temperature below $150{ }^{\circ} \mathrm{C}$.

\section{Results and discussion}

The design of the bi-functional heterogeneous solids bearing both imidazolium-based catalysts and co-catalysts with acid properties was based on a two-step procedure.

In a first step, mesoporous solid supports bearing $\mathrm{Sn}$ and $\mathrm{Zn}$ embedded as single site in the silica structure were successfully synthesized optimizing a previously described procedure (see Experimental section). ${ }^{\mathbf{2 4 2 5}}$ The selected approach allows obtaining particles with reduced size (XS, below $100 \mathrm{~nm}$ ) with a short protocol requiring only $60 \mathrm{~min}$ synthesis time. This range of size was initially selected because of the improved performances of the catalysts displaying a small particle dimension compared to the large particle analogues. ${ }^{26}$ For the sake of comparison, a mesoporous all-silica solid with reduced size (XS-MCM-41) was synthesized as well. Transmission electron microscopy investigation confirmed that both Sn- and Znbased solids (XS-Sn and XS-Zn) as well as XS-MCM-41 display a narrow particle size distribution with a particle diameter comprised between 50 and $100 \mathrm{~nm}$ (Fig. S1†). Moreover, at high magnification, the mesoporous arrangement was also clearly distinguished. The characteristic hexagonal organization of pores was confirmed via low-angle XRD measurements (Fig. S2 $\dagger$ ). All samples display the $d_{100}$ signal at $2 \theta \cong 2^{\circ}$, typical of MCM-41-like architectures. As expected, the main $d_{100}$ reflection was broader than the one observed for MCM-41 with standard dimensions and the additional $d_{110}$ and $d_{200}$ reflections were not clearly visible as a consequence of the reduced size with a consequent decrease of the long range order. ${ }^{27}$ Analysis of the materials via nitrogen physisorption revealed a high specific surface area and a narrow pore size distribution centred at $2.2 \mathrm{~nm}$ (Fig. S3† and Table 1). In view of the selected application, a key parameter is represented by the acidity of the solids, which was investigated by means of adsorption microcalorimetry. In Fig. 1 the differential heat of adsorption, $Q_{\text {diff }}$, is reported as a function of the amount of $\mathrm{NH}_{3}$ adsorbed for both $\mathrm{Sn}-\mathrm{XS}$ and Zn-XS samples. For comparison, the analysis of XSMCM-41 is reported as well. From this investigation emerged

Table 1 Textural properties of the XS-based solids

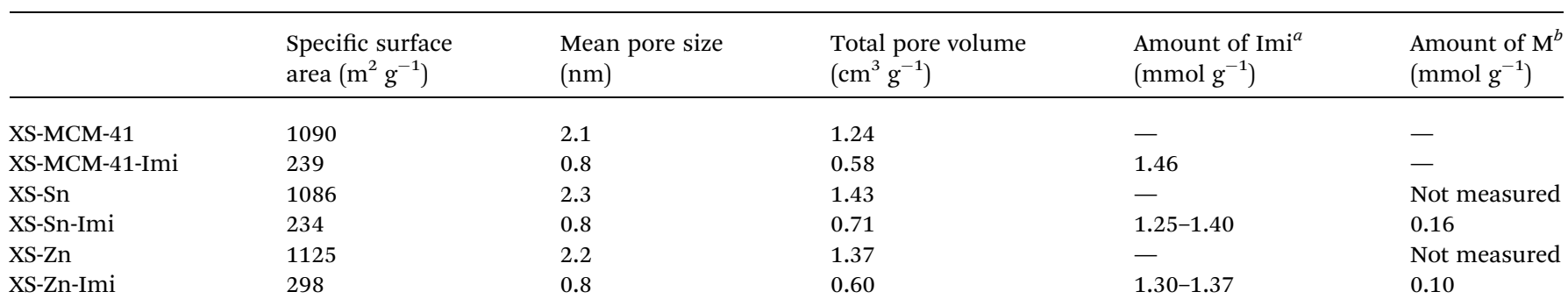

${ }^{a}$ Range of imidazolium salt determined via combustion chemical analysis on different batches. Amount estimated considering the nitrogen percentage. ${ }^{b}$ Amount of metal (M: Sn or Zn) determined via inductively coupled plasma optical emission spectroscopy (ICP-OES). See Experimental section for more details. 


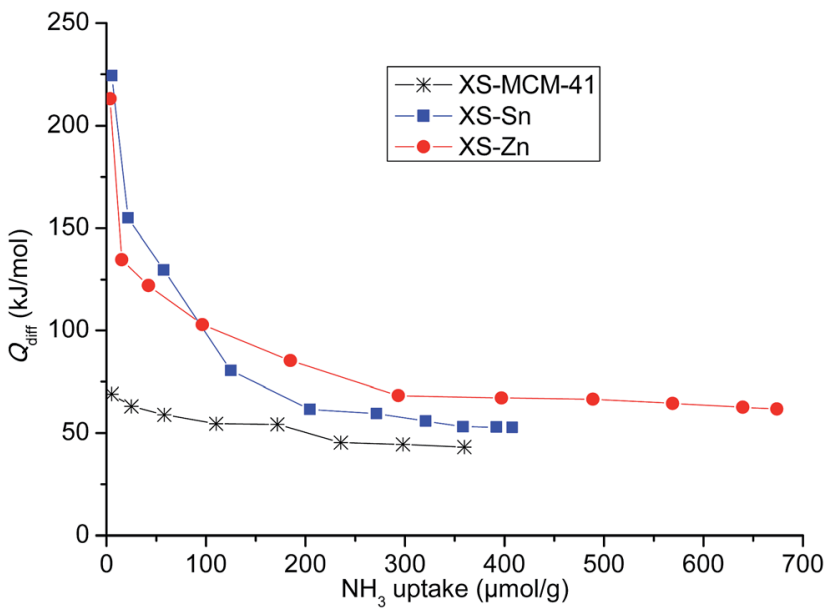

Fig. 1 Differential heat of adsorption $\left(Q_{\text {diff }}\right)$ as a function of the $\mathrm{NH}_{3}$ uptake for XS-Sn, XS-Zn and XS-MCM-41.

that the thermal effect associated with ammonia adsorption on Sn-XS and Zn-XS is much higher than on XS-MCM-41, especially at low $\mathrm{NH}_{3}$ uptake values, indicating that both $\mathrm{Sn}$ - and $\mathrm{Zn}$-based solids display acid properties significantly more pronounced than the all-silica sample. On both Sn-XS and Zn-XS $Q_{\text {diff }}$ is initially higher than $200 \mathrm{~kJ} \mathrm{~mol}^{-1}$, revealing the presence of strong acid sites, and then decreases as the ammonia uptake increases. The point at which the adsorption becomes nonspecific can be established taking into account the results obtained on the pure silica XS-MCM-41 sample, for which differential adsorption heat values as high as $67 \mathrm{~kJ} \mathrm{~mol}^{-1}$ are observed (Fig. 1), in agreement with previous experiments on pure silica samples. ${ }^{28-30}$ Accordingly, for the Sn- and Zn-based samples the fraction of ammonia uptake corresponding to differential heats below $70 \mathrm{~kJ} \mathrm{~mol}^{-1}$ was ascribed to nonspecific and/or physical adsorption on the pure-silica part of the samples, and thus neglected in calculating the acid sites concentration which resulted 170 and $280 \mu \mathrm{mol} \mathrm{g}^{-1}$ for Sn-XS and Zn-XS, respectively. The continuous decrease in the differential heat as the ammonia uptake increases on both samples indicates heterogeneity of the acid sites, which can be either Lewis (the Sn or $\mathrm{Zn}$ centres) or Brønsted sites (bridged $\mathrm{OH}$ groups made acidic by the proximity of the metal site). From the data of ammonia readsorption after overnight evacuation, it was possible to evaluate the differential heat at which the adsorption shifts from irreversible to reversible. It was found that the acid sites able to adsorb ammonia irreversibly are those characterized by $Q_{\text {diff }}$ values higher than $90 \mathrm{~kJ} \mathrm{~mol}^{-1}$, whose concentration is about 110 and $160 \mu \mathrm{mol} \mathrm{g}^{-1}$ for Sn-XS and ZnXS, respectively. From this study emerged that both materials display interesting acid properties in view of their possible application as active supports in the conversion of carbon dioxide.

The second step, requiring the functionalization of the surface with an imidazolium-based catalyst, was performed via a one-pot procedure reacting the previously described XS-solids with an equivalent mixture of 3-chloropropyltrimethoxysilane and 1-methyl imidazole as depicted on scheme 1. This

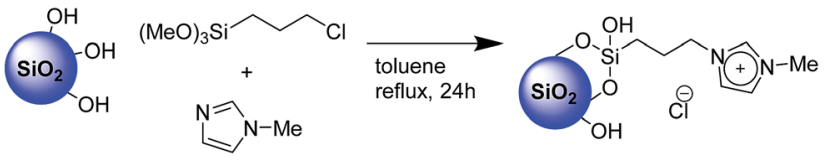

Scheme 1 One-pot surface functionalization of silica with imidazolium salt.

procedure, compared with the standard two-step approach, presents the multiple advantages of a fast functionalization together with a limited number of separation and purification procedures as well as the absence of intermediate characterizations. The amount of organic moieties in the functionalized solids (XS-MCM-41-Imi, XS-Sn-Imi and XS-Zn-Imi) was quantified via combustion chemical analysis (Table 1) while the proof of the presence of imidazolium and not adsorbed imidazole species was obtained via solid state ${ }^{13} \mathrm{C}$-CP-MAS NMR (Fig. 2 and $\mathrm{S} 4 \dagger)^{31,32}$ The anchoring of the trimethoxysilane-based linker to the silica surface was addressed via solid state ${ }^{29} \mathrm{Si}$ NMR performed both in cross polarisation and direct excitation.

The presence of a combination of $\mathrm{T}^{3}$ and $\mathrm{T}^{2}$ signals in the ${ }^{29}$ Si-CP-MAS-NMR (Fig. 3 and S4†े) confirmed that organic moieties were anchored via a covalent bond and not physisorbed on the silica surface.

A deep investigation on the insertion of $\mathrm{Sn}$ and $\mathrm{Zn}$ as single site within the silica scaffold was performed as well. Solid-state ${ }^{119}$ Sn-NMR under static conditions (Fig. 3, left) revealed the presence a broad band centred at $-680 \mathrm{ppm}$ which can be attributed to $\mathrm{Sn}(\mathrm{Iv})$ species in a tetrahedral coordination. ${ }^{33-35}$ The absence of relevant contribution at higher frequencies (around $-600 \mathrm{ppm}$ ) allows excluding the formation of a significant amount of $\mathrm{SnO}_{2}$ due to self-condensation of the tin precursors in the reaction medium. The acquisition of solid ${ }^{67} \mathrm{Zn}$ NMR data was hindered by the intrinsic difficulties of this nucleus. It is known that the low natural abundance of ${ }^{67} \mathrm{Zn}$ together with its large quadrupole moment and the small gyromagnetic ratio represent a highly unfavourable combination of features for solid state nuclear magnetic resonance investigation..$^{36}$ Moreover, the low amount of $\mathrm{Zn}$ inserted in the samples (about $2 \mathrm{wt} \%$ ) reduces also the possibility of efficient use of advanced sensitivity-enhancement techniques or ultrahigh-magnetic-field NMR instruments for a deep investigation of the $\mathrm{Zn}$ coordination sphere. In order to overcome these drawbacks, X-ray photoelectron spectroscopy via analysis

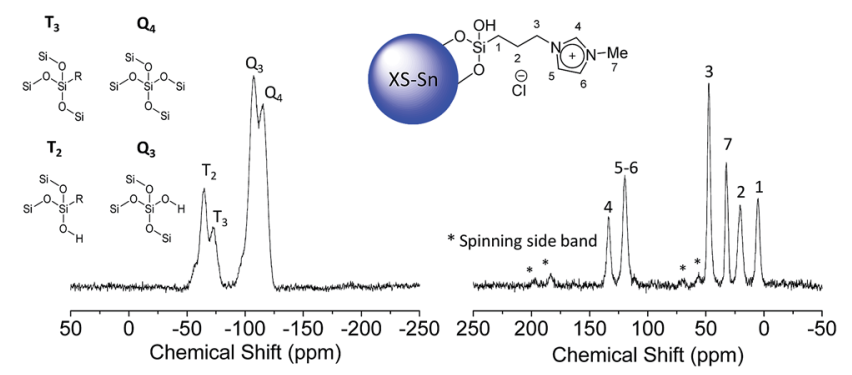

Fig. $2{ }^{29} \mathrm{Si}-\mathrm{CP}-\mathrm{MAS}-\mathrm{NMR}$ (left) and ${ }^{13} \mathrm{C}$-CP-MAS-NMR (right) of XSSn-Imi. 


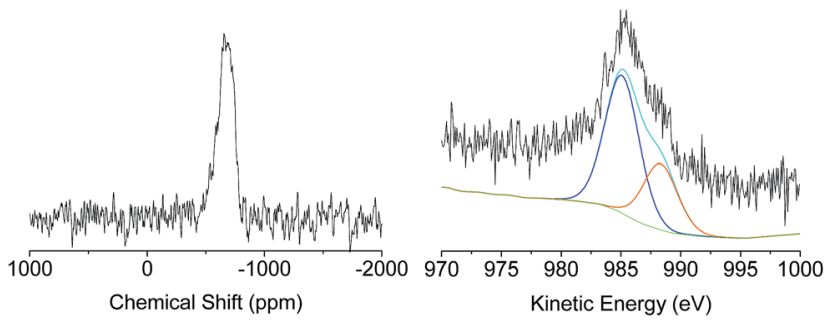

Fig. 3 Solid-state ${ }^{119} \mathrm{Sn}-\mathrm{NMR}$ of XS-Sn (left) and Zn LMM Auger transition of XS-Zn (right)

of the Auger transition (Fig. 3, right) was used to study the insertion of $\mathrm{Zn}$ as single site in the MCM-41 solid. The Zn LMM Auger transition can be considered as an overlapping of two different contributions, the first one centred at $988.08 \mathrm{eV}$ corresponds to extra-framework $\mathrm{ZnO}$ and the second one at $985.30 \mathrm{eV}$ can be attributed to intra-framework $\mathrm{Zn}$ species. ${ }^{24}$ This result indicates that in the case of XS-Zn-Imi the metal centre is partially present ( $c a .30 \%$ of the total amount) in the form of $\mathrm{ZnO}$ extra-framework species.

The structural and textural properties of the final materials (XS-Sn-Imi and XS-Zn-Imi) were investigated via transmission electron microscopy (TEM), X-ray diffraction (XRD) and nitrogen physisorption. TEM analysis of the samples (Fig. 4) revealed that the post-functionalization did not affect the morphology of the materials with aggregation or sintering phenomena. Both XS-Sn-Imi and XS-Zn-Imi display an almost spherical shape and a narrow particle size distribution similar to the non-functionalized samples. As expected, low angle XRD measurements performed on the imidazolium functionalized solids evidenced a pattern similar to the one already observed for XS-Sn and XS-Zn (Fig. S2 $\dagger$ ) confirming that the hexagonal order was preserved during the reaction. As expected, nitrogen physisorption analysis (Fig. 5 and S5 $\dagger$ ) clearly indicated a decrease of the Brunauer-Emmett-Teller (BET) specific surface area as well as of the pore diameter (psd). Due to the shift of the pore size distribution in the microporous region two different methods were applied for the analysis of the data. The standard Barrett-Joyner-Halenda (BJH) was selected to estimate the psd of the non-functionalized XS-solids while the micropore size was evaluated using the method proposed by Horvath and Kawazoe. ${ }^{37,38}$

The variation of the pore diameter suggests that the anchoring of the imidazolium moieties occurs not only at the external surface of the particles but also within the pores. It is

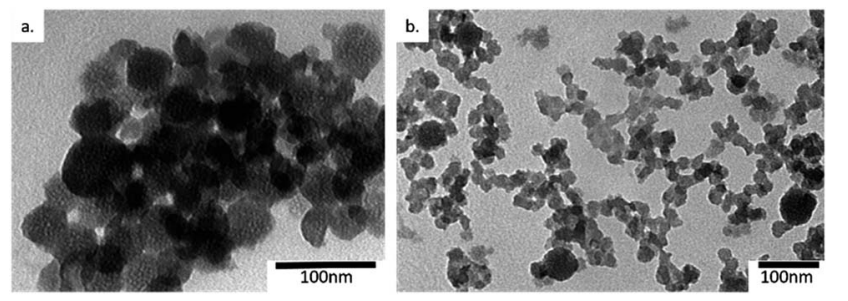

Fig. 4 TEM images of XS-Sn-Imi (a) and XS-Zn-Imi (b).
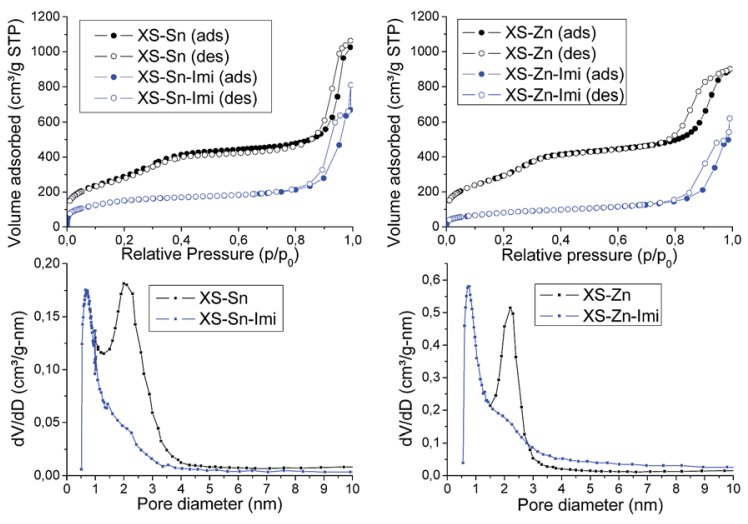

Fig. $5 \quad \mathrm{~N}_{2}$ adsorption-desorption isotherms of XS-Sn and XS-Sn-Imi (up-left) and XS-Zn and XS-Zn-Imi (up-right). Pore size distribution of XS-Sn and XS-Sn-Imi (down-left) and XS-Zn and XS-Zn-Imi (downright).

important to underline that the reduced pore size does not represent a serious drawback in view of the application of these solids in the conversion of carbon dioxide because of the small size of the reactants. As can be seen in Fig. 5 all the materials display a type IV isotherm with an evident hysteresis loop at high relative pressures. This behaviour was previously observed for the materials exhibiting an extra-small size ${ }^{25,26}$ and can be ascribed to the presence of disordered meso-macro pores as consequence of the inter-particle interactions. Both XS-Sn-Imi and XS-Zn-Imi display promising properties for catalytic applications such as high surface area, relevant amount of metal element inserted as single site which provide the desired acidity and good percentage of imidazolium functionalization. Hence, both solids were tested in the reaction of carbon dioxide with epoxides to give the corresponding cyclic carbonates.

A challenging substrate as styrene oxide was selected to evaluate the activity of the catalysts and the reaction temperature was decreased to $125{ }^{\circ} \mathrm{C}$ compared to the standard value (usually this reaction is performed at $150{ }^{\circ} \mathrm{C}$ in absence of cocatalyst)..$^{10,16}$ For comparison, the activity of the all-silica MCM41 solid functionalized with imidazolium moiety (XS-MCM-41Imi) was studied as well (see entry 1 in Table 2). Moreover, before starting the investigation in the presence of the bifunctional catalysts an additional catalytic test adding a small amount of $\mathrm{ZnCl}_{2}$ (corresponding approximatively to the quantity inserted in the silica framework) to the reaction mixture was performed in order to evaluate the positive contribution of a salt with Lewis acid properties under homogeneous conditions. In order to allow a meaningful comparison among the solids, as well as with reference catalysts reported in the literature, two different turnover numbers (TON) were calculated. This choice is justified by the presence of two different catalytic active species behaving as catalyst (imidazolium moieties) and cocatalysts (metal inserted as single site) both displaying a synergic role in the reaction. The TON considering the catalyst $\left(\mathrm{TON}_{\mathrm{imi}}\right)$ was defined as moles of epoxide converted/moles of imidazolium salt while the TON based on the co-catalyst $\left(\mathrm{TON}_{\mathrm{M}}\right)$ was defined as moles of epoxide converted/moles of Sn or Zn. 
Table 2 Conversion of styrene oxide into the corresponding cyclic carbonate with use of different catalysts ${ }^{a}$

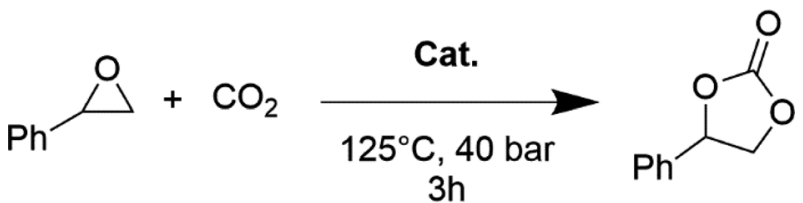

\begin{tabular}{|c|c|c|c|c|c|c|c|}
\hline Entry & Catalyst & Amount $(\mathrm{g})$ & EtOH $(\mathrm{mL})$ & Conversion (\%) & Selectivity (\%) & $\mathrm{TON}_{\mathrm{imi}}{ }^{b}$ & $\mathrm{TON}_{\mathrm{M}}{ }^{c}$ \\
\hline 1 & XS-MCM-41-Imi & 1 & 1.5 & 54 & $>95$ & 78 & - \\
\hline 2 & $\mathrm{XS}^{-M C M}-41-$ Imi $^{d}$ & 1 & 1.5 & 63 & $>95$ & 91 & 901 \\
\hline 4 & XS-Sn-Imi & 0.5 & 1.5 & 37 & $>95$ & 116 & 948 \\
\hline 5 & XS-Zn-Imi & 0.5 & 1.5 & 39 & $>95$ & 127 & 1489 \\
\hline 6 & XS-Sn-Imi & 0.5 & - & 32 & $>95$ & 96 & 826 \\
\hline
\end{tabular}

${ }^{a}$ Reaction conditions: styrene oxide $(24.0 \mathrm{~mL} ; 210 \mathrm{mmol}), \mathrm{CO}_{2}$ pressure $(40 \mathrm{bar})$, temperature $\left(125{ }^{\circ} \mathrm{C}\right), 500 \mathrm{rpm} .{ }^{b}$ Turnover number $(\mathrm{TON})$ calculated as moles of epoxide converted/moles of imidazolium sites. Amount of imidazolium salt quantified via chemical combustion analysis for each catalyst. ${ }^{c}$ Turnover number (TON) calculated as moles of epoxide converted/moles of Sn or Zn. Amount of metal (Sn or Zn) quantified

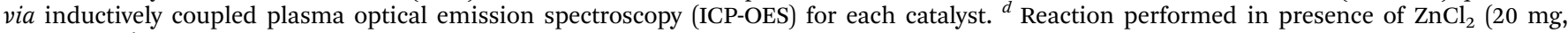
$0.147 \mathrm{mmol})$.

As expected, the presence of $\mathrm{ZnCl}_{2}$ caused an improvement of the catalytic performances of the XS-MCM-Imi solid from $54 \%$ to $63 \%$ conversion (compare entries 1 and 2 in Table 2). Interestingly in the presence of XS-Sn-Imi similar performances compared to the homogeneous Lewis acid catalyst were obtained. The activity of the XS-Sn-Imi solid was preserved after decreasing the amount of catalyst (Table 2, entries 3 and 4). The slightly increase of $\mathrm{TON}_{\mathrm{imi}}$ obtained under the conditions reported in entry 4 could be attributed to a better dispersion of the solid in the reaction mixture. XS-Zn-Imi catalyst allowed achieving similar conversion (compare entries 4 and 5 in Table 2 ), thus further proving the positive influence of the insertion of single sites within the silica structure.

All the previously described experiments were performed under the same reaction conditions, hence in presence of a small amount $(1.5 \mathrm{~mL})$ of ethanol needed to solubilize the $\mathrm{ZnCl}_{2}$ added in the initial test. However, since it is known that the presence of hydroxyl groups in the reaction mixture may also facilitate the opening of the epoxide via formation of hydrogen bonds, analogous experiments in the absence of alcohol were performed as well.

No relevant differences were observed for both XS-Sn-Imi and XS-Zn-Imi catalysts (compare entries 4 with 6 and 5 with 7), thus proving that the small amount of ethanol was not the main responsible of the increased activity and further confirming the positive active role of the XS-Sn and XS-Zn supports.

The better performances of XS-Zn-Imi in terms of $\mathrm{TON}_{\mathrm{M}}$ could be attributed to the higher acidity of the support.

The versatility of the catalysts was checked selecting the most challenging (slightly less performant) XS-Sn-imi solid. The data concerning the activity of the Sn-based material in the presence of various epoxides are reported in Table 3. From this investigation emerged that the XS-Sn-Imi displays excellent performances in the presence of various epoxides including the extremely low reactive cyclohexene oxide. Moreover, in the presence of epichlorohydrin and glycidol excellent performances were achieved at the challenging temperatures of $80{ }^{\circ} \mathrm{C}$ and $60{ }^{\circ} \mathrm{C}$ respectively (entries 7 and 9). All these results confirm the positive synergic effect of the catalyst and co-catalyst.

To challenge even more the XS-Sn-Imi, a test with glycidol at $30{ }^{\circ} \mathrm{C}$ (entry 10) was performed as well. Also under these conditions, the catalyst showed a good $\mathrm{TON}_{\mathrm{imi}}$, higher than other bi-functional catalysts reported in the literature employed under homogeneous ${ }^{39}\left(\mathrm{TON}_{\mathrm{imi}}\right.$ of 26$)$ or heterogeneous conditions in the presence of the same epoxide.

In the recent literature, many efforts were dedicated to the decrease of the working temperature and/or pressure (e.g. using MOF). However, this goal is usually achieved employing imidazolium or more often ammonium salts in homogeneous phase. ${ }^{\mathbf{4 0 - 4 5}}$ It should be mentioned that a direct comparison with the literature is hampered by the huge differences in the reaction conditions employed for the synthesis of cyclic carbonates including presence of solvent, substrate to catalyst molar ratio, nature of nucleophile, use of co-catalyst, temperature, pressure and reaction time. These difficulties can be partially reduced if the catalysts are compared in terms of turnover number or turnover frequency (TOF defined as TON/ time). Our solids display better $\mathrm{TON}_{\mathrm{imi}}$ than some of the most active catalysts previously reported in the literature and used in the absence of co-catalyst. Moreover, they also display excellent $\mathrm{TON}_{\mathrm{M}}$ or $\mathrm{TOF}_{\mathrm{M}}$ if compared with other bi-functional heterogeneous catalysts. One of the best comparison can be probably done with similar silica-based porous materials embedding titanium as single site $e^{\mathbf{4 6}}$ and tested in the presence of styrene oxide at $120{ }^{\circ} \mathrm{C}$ under solvent free conditions which displayed $\mathrm{TON}_{\mathrm{M}}$ much lower than the ones achieved with both XS-Sn-Imi and XS-Zn-Imi. Other metal-free catalysts containing only imidazolium-based species can be also selected for comparison to evidence the importance of the co-catalysts. One of the most active silica-supported imidazolium salts displayed a $\mathrm{TON}_{\mathrm{imi}}$ of 
Table 3 Conversion of various epoxides into corresponding cyclic carbonates with use of XS-Sn-Imi as catalyst ${ }^{a}$

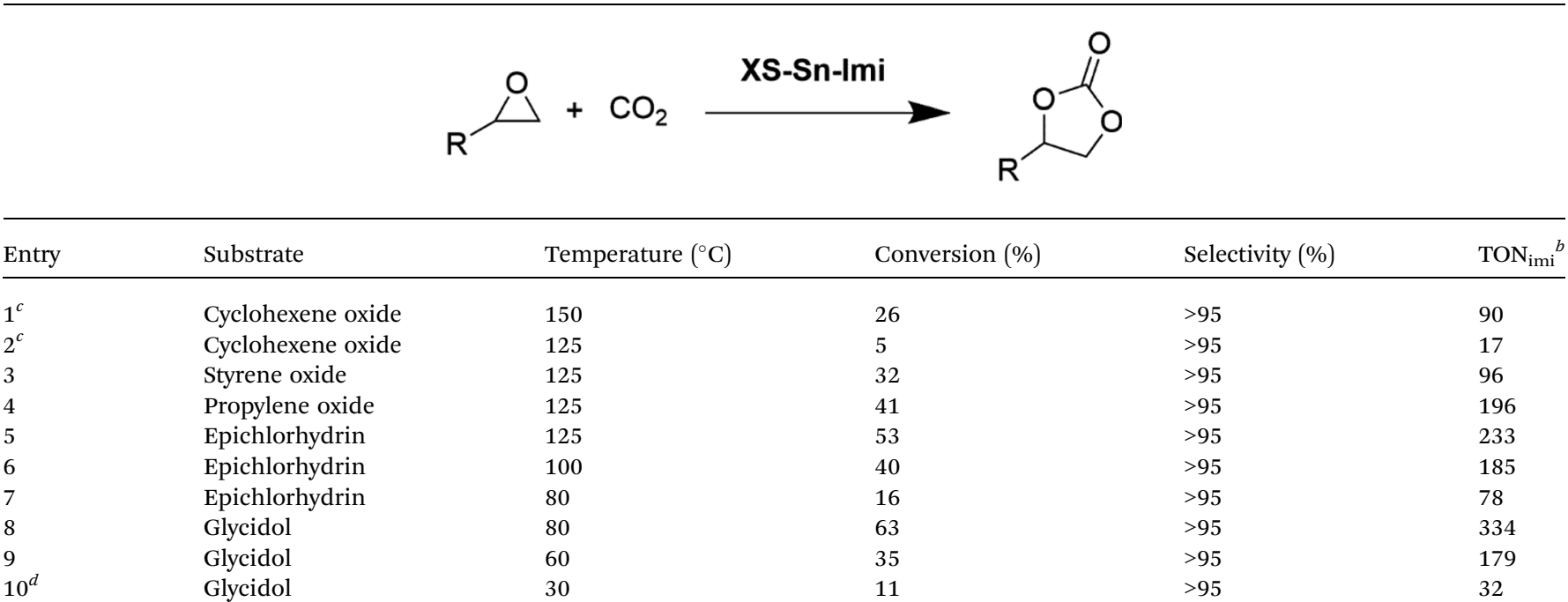

${ }^{a}$ Reaction conditions: epoxide $(24.0 \mathrm{~mL})$, catalyst $(500 \mathrm{mg}), \mathrm{CO}_{2}$ pressure $(40 \mathrm{bar}), 500 \mathrm{rpm} .{ }^{b}$ Turnover number (TON $\left.\mathrm{imi}\right)$ calculated as moles of epoxides converted/moles of imidazolium active sites. Amount of imidazolium salts quantified via chemical combustion analysis for each catalyst (not all the catalyst used comes from the same preparation). ${ }^{c}$ Reaction performed for $24 \mathrm{~h} .{ }^{d}$ Reaction performed for $6 \mathrm{~h}$ with $1 \mathrm{~g}$ of catalyst.

88 in presence of styrene oxide and under conditions similar to those used for the present work. However, it deserves to be mentioned that the counter-anion/nucleophile used was the more active iodide. ${ }^{47}$

Still considering the synthesis of styrene carbonate, similar TON (e.g. 101 and 106) were achieved with other structured supports such as carbon nanohorns but these catalysts required higher temperature $\left(150{ }^{\circ} \mathrm{C}\right) .{ }^{17}$

When working under heterogeneous conditions, the excellent catalytic performances of a solid are not the only parameter to be considered, the true heterogeneous nature of the catalyst should be also evaluated. In order to exclude the possible leaching of active species in solution and confirm the stability of catalyst under the selected reactions conditions, recycle experiments were performed (Fig. 6). After each cycle, the XS-SnImi catalyst was recovered from the reaction mixture by centrifugation, washed and reused (see Experimental section

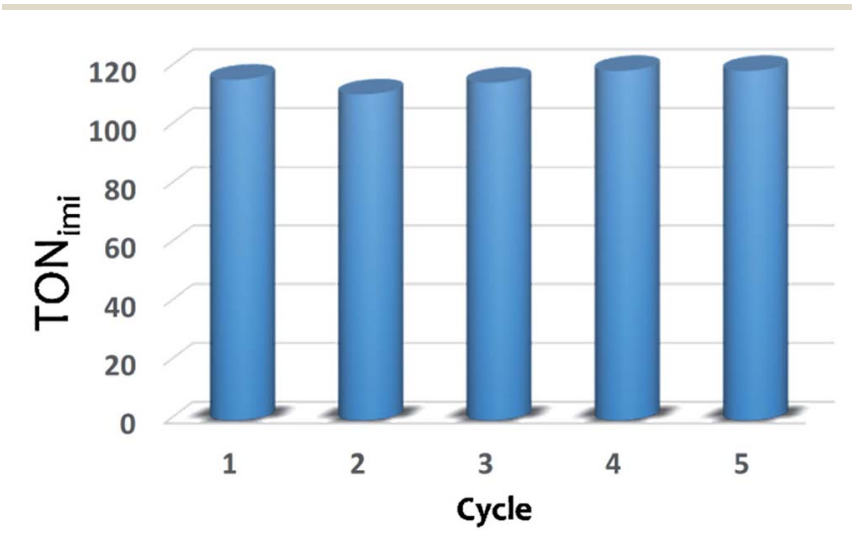

Fig. 6 Catalytic performance of XS-Sn-Imi in subsequent reaction cycles. After each cycle, the solid was washed several times with toluene and ethanol, dried and reused without additional treatments. for more details) without any additional activation treatment. The data reported in Fig. 6 evidence that the catalyst can be used in multiple cycles without any decrease in the catalytic performances.

\section{Experimental section}

\section{Materials and methods}

Ammonium hydroxide (30\%) was purchased from Carl Roth. Cetyltrimethylammonium bromide (CTAB), tetraethylorthosilicate (TEOS), $N$-methylimidazole, (3-chloropropyl)trimethoxysilane, styrene oxide, epichlorohydrin, cyclohexene oxide, propylene oxide were purchased from TCI. $\mathrm{SnCl}_{4} \cdot 5 \mathrm{H}_{2} \mathrm{O}$, $\mathrm{Zn}\left(\mathrm{NO}_{3}\right)_{2} \cdot 6 \mathrm{H}_{2} \mathrm{O}$, glycidol were purchased from Sigma-Aldrich. All chemicals were used as received without any further purification.

Transmission electron microscopy (TEM) images were obtained using a Philips Tecnai 10 microscope operating at $80 \mathrm{kV}$. Samples were prepared by dispersion of a small quantity of material in absolute ethanol and deposited onto a copper grid. Nitrogen adsorption-desorption analyses were carried out at 77 $\mathrm{K}$ with a volumetric adsorption analyser (Micromeritics Tristar 3000 and ASAP 2000). Prior to the analysis, the samples were pre-treated at $150{ }^{\circ} \mathrm{C}$ for $16 \mathrm{~h}$ under reduced pressure $(0.1$ mbar). The Brunauer-Emmett-Teller (BET) method was applied in the $0.05-0.30 \mathrm{p} / \mathrm{p}_{0}$ range to calculate the specific surface area, while the pore size distributions were calculated from the adsorption isotherm using the Barrett-Joyner-Halenda (BJH) method. Powder X-ray diffraction (XRD) patterns were recorded on a PANalytical X'pert diffractometer with $\mathrm{Cu} \mathrm{K} \alpha$ radiation $(\lambda=$ $1.54178 \AA$ A). A Tian-Calvet heat flow calorimeter (Setaram) equipped with a volumetric vacuum line was used for microcalorimetric measurements. Each sample was pre-treated overnight at $250{ }^{\circ} \mathrm{C}$ under vacuum $\left(10^{-3} \mathrm{~Pa}\right)$. Ammonia 
adsorption was carried out at $80{ }^{\circ} \mathrm{C}$ by admitting successive doses of the probe gas and recording the corresponding thermal effect. The run was stopped at a final pressure of 133.3 Pa. After overnight outgassing at $80{ }^{\circ} \mathrm{C}$, a second adsorption run was carried out. The Si environment and the coordination of the Sn atoms were studied by ${ }^{29} \mathrm{Si}$ Magic Angle Spinning (MAS) and static ${ }^{119} \mathrm{Sn}$ Nuclear Magnetic Resonance (NMR). ${ }^{29} \mathrm{Si}$ and ${ }^{13} \mathrm{C}$ NMR spectra were recorded at room temperature on a Bruker Avance-500 spectrometer operating at 11.7 T (99.3 MHz for ${ }^{29} \mathrm{Si}$ and $125.7 \mathrm{MHz}$ for ${ }^{13} \mathrm{C}$ ) using a $4 \mathrm{~mm}$ CP-MAS Bruker probe. The sample was packed in a $4 \mathrm{~mm}$ zirconia rotor and measured with a spinning frequency of $8000 \mathrm{~Hz}$. Cross polarization CPMAS spectra were recorded using a $5 \mathrm{~s}$ relaxation delay and 5 $\mathrm{ms}$ contact time for ${ }^{29} \mathrm{Si}$ and $2 \mathrm{~ms}$ for ${ }^{13} \mathrm{C}$. The processing comprised exponential multiplication of the FID with a line broadening factor of $30 \mathrm{~Hz}$ for ${ }^{29} \mathrm{Si}$ or $10 \mathrm{~Hz}$ for ${ }^{13} \mathrm{C}$, zerofilling, Fourier transform, phase and baseline corrections. The chemical shift scale was calibrated at room temperature with respect to a sample of solid 3-(trimethylsilyl)-1-propanesulfonic acid sodium salt (DSS) (0.0 ppm). ${ }^{119} \mathrm{Sn}$ NMR spectra were recorded at room temperature on a Varian VNMRS-400 spectrometer operating at $9.4 \mathrm{~T}\left(149 \mathrm{MHz}\right.$ for $\left.{ }^{119} \mathrm{Sn}\right)$ using a $10 \mathrm{~mm}$ wideline probe. The sample was packed in a $10 \mathrm{~mm}$ Kel-F container and studied in static condition. ${ }^{119} \mathrm{Sn}$ spectra were recorded using the Hahn echo pulse sequence and following acquisition parameters: $60 \mathrm{~s}$ relaxation delay, $3.0 \mu \mathrm{s}\left(90^{\circ}\right)$ excitation pulse, 5 ms acquisition time. The processing comprised exponential multiplication of the FID with a line broadening factor of $1000 \mathrm{~Hz}$, zero-filling, Fourier transform, phase and baseline corrections. The chemical shift scale was calibrated at room temperature with respect to the isotropic shift of $\mathrm{SnO}_{2}$ to -603 ppm. ${ }^{48}{ }^{1} \mathrm{H}$ NMR spectroscopy of liquid samples was performed on a JEOL ECA operating at 9.4 $\mathrm{T}(400 \mathrm{MHz})$. The X-ray photoelectron spectroscopy (XPS) analyses were performed on a ThermoFisher ESCALAB 250Xi instrument. Inductively coupled plasma optical emission spectroscopy (ICP-OES) employed to determine the chemical composition of the materials was performed an Optima 8000 ICP-OES Spectrometer.

Chemical combustion analyses were performed on a PerkinElemer 2400 Serie 2 analyser.

\section{Catalysts preparation}

General procedure for the synthesis of XS-MCM-41, XS-Sn and XS-Zn solids. Silica based materials were prepared according a dilute route. ${ }^{24}$ Ammonium hydroxide $(1.42 \mathrm{~mL}$, $10.95 \mathrm{mmol})$ and CTAB (1.52 g, $4.16 \mathrm{mmol})$ were added to $726 \mathrm{~mL}$ of Milli-Q water. After 30 minutes under agitation, TEOS $(6.936 \mathrm{~g}, 33.29 \mathrm{mmol})$ and the proper amount of $\mathrm{SnCl}_{4} \cdot 5 \mathrm{H}_{2} \mathrm{O}$ $(157.6 \mathrm{mg}, 0.45 \mathrm{mmol})$ or $\mathrm{Zn}\left(\mathrm{NO}_{3}\right)_{2} \cdot 6 \mathrm{H}_{2} \mathrm{O}$ (133.9 mg, 0.450 $\mathrm{mmol}$ ) previously dissolved in $2 \mathrm{~mL}$ of absolute ethanol were added dropwise in the aqueous solution. After $1 \mathrm{~h}$ under agitation at room temperature, the solid was filtered and washed 3 times alternatively with water and ethanol. The solid was dried overnight and calcined under air for $8 \mathrm{~h}$ at $550{ }^{\circ} \mathrm{C}$ with heating and cooling rate of $2{ }^{\circ} \mathrm{C} \min ^{-1}$.
Synthesis of XS-MCM-41-Imi, XS-Sn-Imi and XS-Zn-Imi. XSMCM-41, XS-Sn and XS-Zn were dried in oven at $100{ }^{\circ} \mathrm{C}$ overnight before functionalisation. In a round bottom flask, the XSsolid (1.5 g) was suspended in $15 \mathrm{~mL}$ of dry toluene. $N$-Methylimidazole $(24 \mathrm{mmol})$ and (3-chloropropyl)trimethoxysilane (12 mmol) were added. The mixture was stirred at reflux temperature for $24 \mathrm{~h}$. After cooling, the functionalised solid was washed with Soxhlet extractor using methanol as solvent. The amount of imidazolium was evaluated through chemical combustion analysis.

\section{Catalytic tests}

All catalytic tests were performed in a Cambridge Design Bullfrog batch reactor with individual control of the temperature and the stirring. During the entire experiment in addition to temperature and stirring, the evolution of pressure can be monitored as well. Prior to any experiment, the catalyst was dried overnight at $100{ }^{\circ} \mathrm{C}$. In a typical test, the catalyst was added to the epoxide $(24.0 \mathrm{~mL})$ in a Teflon vial under solventfree conditions. After closing the reactor, the mixture was stirred at $500 \mathrm{rpm}$. The system was purged with a gentle flow of $\mathrm{N}_{2}$ before the addition of $\mathrm{CO}_{2}$. After a short period of pressure stabilisation at room temperature, the system was heated to the desired temperature with a rate of $1^{\circ} \mathrm{C} \mathrm{min}^{-1}$. The reaction was allowed to run at the desired temperature for a variable time (in function of the selected epoxide). After this time, the reactor was cooled down to room temperature, the pressure was released and the reactor was opened. The reaction mixture was submitted to centrifugation for $10 \mathrm{~min}$ at $4500 \mathrm{rpm}$. The supernatant was removed and analysed by ${ }^{1} \mathrm{H}-\mathrm{NMR}$ using $\mathrm{CDCl}_{3}$ as solvent.

For recycling experiment, the solid was added in the centrifugation tube in presence of toluene $(50 \mathrm{~mL})$ and it was sonicated for 15 minutes, centrifuged at $4500 \mathrm{rpm}$ for $10 \mathrm{~min}$ and the supernatant removed. This washing operation was repeated 2 times with toluene and 2 times with ethanol. Then the solid was dried at $100{ }^{\circ} \mathrm{C}$ overnight prior to next catalytic run.

\section{Conclusion}

Two bi-functional silica-based materials bearing tin or zinc inserted as single sites within the silica architecture acting as acid centres and decorated with imidazolium moieties as the nucleophile source (XS-Sn-Imi and XS-Zn-Imi) were successfully synthesized. The solids display promising features for catalytic applications including high surface area, reduced particle size, good amount of imidazolium salts and acid properties estimated via microcalorimetry analysis. Both XS-Sn-Imi and XS-ZnImi were used as catalyst for the chemical fixation of carbon dioxide onto epoxides to give the corresponding cyclic carbonates, displaying excellent performances. The versatility of the Sn-based catalyst was proved in the presence of various epoxides. In all experiments, the bi-functional solids allow reduction of the reaction temperature below $150{ }^{\circ} \mathrm{C}$. Moreover, with glycidol the temperature was decreased down to $30{ }^{\circ} \mathrm{C}$. The short synthesis protocol of the heterogeneous catalysts, together with 
the $100 \%$ atom economy of the target reaction and the low reaction temperature make the entire process highly sustainable. The XS-Sn-Imi catalyst was stable under the selected reaction conditions and reusable for multiple catalytic cycles.

\section{Conflicts of interest}

There are no conflicts to declare.

\section{Acknowledgements}

A. Comès acknowledges the University of Namur for a $\mathrm{PhD}$ fellowship. The authors acknowledge FNRS for supporting this work. This research used resources of the "Plateforme Technologique Physico-Chemical Characterisation" - PC2. This research also used resources of the Electron Microscopy Service located at the University of Namur. This Service is member of the "Plateforme Technologique Morphologie - Imagerie".

\section{Notes and references}

1 C. Maeda, Y. Miyazaki and T. Ema, Catal. Sci. Technol., 2014, 4, 1482-1497.

2 G. Fiorani, W. Guo and A. W. Kleij, Green Chem., 2015, 17, 1375-1389.

3 D. Darensbourg, Coord. Chem. Rev., 1996, 153, 155-174.

4 M. North, R. Pasquale and C. Young, Green Chem., 2010, 12, 1514-1539.

5 A. M. Chapman, C. Keyworth, M. R. Kember, A. J. J. Lennox and C. K. Williams, ACS Catal., 2015, 5, 1581-1588.

6 P. P. Pescarmona and M. Taherimehr, Catal. Sci. Technol., 2012, 2, 2169-2187.

7 M. R. Kember, A. Buchard and C. K. Williams, Chem. Commun., 2011, 47, 141-163.

8 F. Jutz, J. M. Andanson and A. Baiker, Chem. Rev., 2011, 111, 322-353.

9 C. Aprile, H. Garcia and P. P. Pescarmona, in Catalytic Methods in Asymmetric Synthesis, John Wiley \& Sons, Inc., 2011, pp. 177-208, DOI: 10.1002/9781118087992.ch3.

10 M. Buaki-Sogo, H. Garcia and C. Aprile, Catal. Sci. Technol., 2015, 5, 1222-1230.

11 L. A. Bivona, O. Fichera, L. Fusaro, F. Giacalone, M. BuakiSogo, M. Gruttadauria and C. Aprile, Catal. Sci. Technol., 2015, 5, 5000-5007.

12 H. Zhong, Y. Su, X. Chen, X. Li and R. Wang, ChemSusChem, 2017, 10, 4855-4863.

13 T. Wang, W. Wang, Y. Lyu, X. Chen, C. Li, Y. Zhang, X. Song and Y. Ding, RSC Adv. , 2017, 7, 2836-2841.

14 S. Baj, T. Krawczyk, K. Jasiak, A. Siewniak and M. Pawlyta, Appl. Catal., A, 2014, 488, 96-102.

15 L. Han, H. Li, S.-J. Choi, M.-S. Park, S.-M. Lee, Y.-J. Kim and D.-W. Park, Appl. Catal., A, 2012, 429-430, 67-72.

16 M. Buaki-Sogó, A. Vivian, L. A. Bivona, H. García, M. Gruttadauria and C. Aprile, Catal. Sci. Technol., 2016, 6, 8418-8427.
17 C. Calabrese, L. F. Liotta, E. Carbonell, F. Giacalone, M. Gruttadauria and C. Aprile, ChemSusChem, 2017, 10, 1202-1209.

18 T. Sakakura, J. C. Choi and H. Yasuda, Chem. Rev., 2007, 107, 2365-2387.

19 F. Ahmadi, S. Tangestaninejad, M. Moghadam, V. Mirkhani, I. Mohammadpoor-Baltork and A. R. Khosropour, Inorg. Chem. Commun., 2011, 14, 1489-1493.

20 M. V. Escarcega-Bobadilla, M. Martinez Belmonte, E. Martin, E. C. Escudero-Adan and A. W. Kleij, Chemistry, 2013, 19, 2641-2648.

21 W. Y. Gao, Y. Chen, Y. Niu, K. Williams, L. Cash, P. J. Perez, L. Wojtas, J. Cai, Y. S. Chen and S. Ma, Angew. Chem., Int. Ed. Engl., 2014, 53, 2615-2619.

22 J. Liang, Y. Q. Xie, Q. Wu, X. Y. Wang, T. T. Liu, H. F. Li, Y. B. Huang and R. Cao, Inorg. Chem., 2018, 57, 2584-2593.

23 M. Ding and H.-L. Jiang, ACS Catal., 2018, 3194-3201, DOI: 10.1021/acscatal.7b03404.

24 X. Collard, P. Louette, S. Fiorilli and C. Aprile, Phys. Chem. Chem. Phys., 2015, 17, 26756-26765.

25 L. Li, X. Collard, A. Bertrand, B. F. Sels, P. P. Pescarmona and C. Aprile, J. Catal., 2014, 314, 56-65.

26 X. Collard, L. Li, W. Lueangchaichaweng, A. Bertrand, C. Aprile and P. P. Pescarmona, Catal. Today, 2014, 235, 184-192.

27 N. Godard, X. Collard, A. Vivian, L. A. Bivona, S. Fiorilli, L. Fusaro and C. Aprile, Appl. Catal., A, 2018, 556, 73-80.

28 N. Cardona-Martinez and J. A. Dumesic, J. Catal., 1990, 125, 427-444.

29 G. Colón, I. Ferino, E. Rombi, E. Selli, L. Forni, P. Magnoux and M. Guisnet, Appl. Catal., A, 1998, 168, 81-92.

30 D. Meloni, D. Perra, R. Monaci, M. G. Cutrufello, E. Rombi and I. Ferino, Appl. Catal., B, 2016, 184, 163-173.

31 N. Fattori, C. M. Maroneze, L. P. da Costa, M. Strauss, F. A. Sigoli, I. O. Mazali and Y. Gushikem, Langmuir, 2012, 28, 10281-10288.

32 L. Wang and E. Y. X. Chen, ACS Catal., 2015, 5, 6907-6917. 33 N. Godard, A. Vivian, L. Fusaro, L. Cannavicci, C. Aprile and D. P. Debecker, ChemCatChem, 2017, 9, 2211-2218.

34 P. Shah, A. V. Ramaswamy, K. Lazar and V. Ramaswamy, Microporous Mesoporous Mater., 2007, 100, 210-226.

35 E. V. Beletskiy, X. Hou, Z. Shen, J. R. Gallagher, J. T. Miller, Y. Wu, T. Li, M. C. Kung and H. H. Kung, J. Am. Chem. Soc., 2016, 138, 4294-4297.

36 S. E. Ashbrook and S. Sneddon, J. Am. Chem. Soc., 2014, 136, 15440-15456.

37 G. Horvath and K. Kawazoe, J. Chem. Eng. Jpn., 1983, 16, 470475.

38 P. L. Llewellyn, E. Bloch and S. Bourrelly, in Characterization of Solid Materials and Heterogeneous Catalysts, Wiley-VCH Verlag GmbH \& Co. KGaA, 2012, pp. 853-879, DOI: 10.1002/9783527645329.ch19.

39 J. Melendez, M. North and P. Villuendas, Chem. Commun., 2009, 2577-2579, DOI: 10.1039/b900180h.

40 M. Taherimehr, B. Van de Voorde, L. H. Wee, J. A. Martens, D. E. De Vos and P. P. Pescarmona, ChemSusChem, 2017, 10, 1283-1291. 
41 J. Lan, M. Liu, X. Lu, X. Zhang and J. Sun, ACS Sustainable Chem. Eng., 2018, 6, 8727-8735.

42 X. Guo, Z. Zhou, C. Chen, J. Bai, C. He and C. Duan, ACS Appl. Mater. Interfaces, 2016, 8, 31746-31756.

43 Z. Xue, J. Jiang, M.-G. Ma, M.-F. Li and T. Mu, ACS Sustainable Chem. Eng., 2017, 5, 2623-2631.

44 J. Song, Z. Zhang, S. Hu, T. Wu, T. Jiang and B. Han, Green Chem., 2009, 11, 1031-1036.

45 Y. Ren, Y. Shi, J. Chen, S. Yang, C. Qi and H. Jiang, RSC Adv., 2013, 3, 2167-2170.
46 D. Srinivas and P. Ratnasamy, Microporous Mesoporous Mater., 2007, 105, 170-180.

47 P. Agrigento, S. M. Al-Amsyar, B. Sorée, M. Taherimehr, M. Gruttadauria, C. Aprile and P. P. Pescarmona, Catal. Sci. Technol., 2014, 4, 1598-1607.

48 C. Cossement, J. Darville, J.-M. Gilles, J. B. Nagy, C. Fernandez and J.-P. Amoureux, Magn. Reson. Chem., 1992, 30, 263-270. 